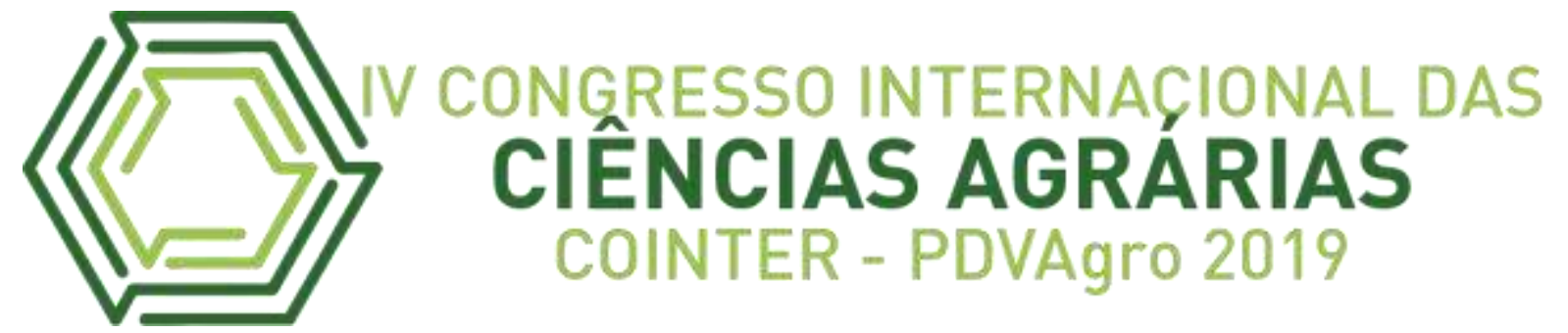

\title{
COMPARAÇÃO DE MODELOS PREDITIVOS PARA O NÍVEL DE RADIAÇÃO NO MUNICÍPIO DE GARANHUNS
}

\author{
COMPARACIÓN DE MODELOS PREDICTIVOS PARA EL NIVEL DE \\ RADIACIÓN EN LA CIUDAD DE GUARANHUNS
}

\section{COMPARISON OF PREDICTIVE MODELS FOR RADIATION LEVEL IN GARANHUNS CITY}

\author{
Apresentação: Comunicação Oral \\ Ruben Vivaldi Silva Pessoa ${ }^{1}$; Jucarlos Rufino de Freitas ${ }^{2}$; Joelma Mayara da Silva ${ }^{3}$ Mickaelle \\ Maria Almeida Pereira ${ }^{4}$; Moacyr Cunha Filho ${ }^{5}$
}

DOI: https://doi.org/10.31692/2526-7701.IVCOINTERPDVAgro.2019.0163

\begin{abstract}
Resumo
Buscando analisar de forma abrangente os dados de radiação dos últimos dez anos no município de Garanhuns, foram ajustados modelos de series temporais dentre os classificados como mais indicados, levando em consideração não só a natureza das informações presentes neste estudo, como também o interesse particular por predizer os níveis de radiação para os próximos anos na localidade, de forma precisa e leal aos dados apresentados. Ao selecionar os modelos que melhor se adequariam aos propósitos do trabalho, foram realizados testes e análises comparativas a fim de encontrar o que apresentava melhor ajuste e consequentemente, o que possuía maior capacidade de realizar uma previsão para os níveis de radiação no período estipulado em Garanhuns. Na pesquisa, foram utilizados dados dos níveis de radiação no município por dia para o ciclo em análise, e ainda informações acerca de variáveis como a umidade do ar, a temperatura, características do vento (direção, rajada e velocidade), dentre outras, sendo todas disponibilizadas pelo Instituto Nacional de Meteorologia (INMET). Para análise comparativa entre os modelos, foram utilizados os testes de Dicker-Fuller Aumentado, Shapiro-Wilk, Kruskal-Wallis, visando verificar a estacionariedade, normalidade e sazonalidade, respectivamente, para os modelos em questão. Também, os tipos de modelos que estiveram em comparação foram o ARIMA e o ARIMAX, que originalmente são semelhantes, o que reforça a importância da aplicação correta dos testes e análises comparativas. Foi constatado que o melhor modelo a ser aplicado é o ARIMAX, que identifica bem os ciclos de crescimento e decrescimento dos níveis de radiação durante o período estudado. Quanto à previsão, para os próximos dez anos, é esperado que mesmo com
\end{abstract}

\footnotetext{
${ }^{1}$ Programa de Pós-Graduação em Biometria e Estatística Aplicada, UFRPE, rvsp1@de.ufpe.br 2Programa de Pós-Graduação em Biometria e Estatística Aplicada, UFRPE, jucarlos123@hotmail.com ${ }^{3}$ Programa de Pós-Graduação em Biometria e Estatística Aplicada, UFRPE, jms.estat@gmail.com ${ }^{4}$ Programa de Pós-Graduação em Biometria e Estatística Aplicada, UFRPE, mickaellealmeida1@gmail.com ${ }^{5}$ Phd, UFRPE, moacyr2006@gmail.com
} 
a ocorrência do comportamento periódico, a variabilidade deve aumentar. Por fim, concluiuse que a utilização do modelo ARIMAX contribui para prevenção de doenças e para um uso mais eficiente e inteligente da energia solar em Garanhuns.

Palavras-Chave:Ajuste, Predição, Radiação, Garanhuns.

\title{
Resumen
}

Buscando analizar de forma comprensiva los datos de radiación de los últimos diez años en el municipio de Garanhuns, se ajustaron los modelos de series temporales clasificándolas como las más indicadas, teniendo en cuenta no solo la naturaleza de la información presente en este estudio, sino también el interés particular por predecir los niveles de insolación para los próximos años en la localidad, con precisión y fidelidad a los datos presentados. Al seleccionar los modelos que mejor se adaptaría al propósito del trabajo, se realizaron análisis comparativas y pruebas para encontrar el que presentaba el mejor ajuste y, en consecuencia, el que tenía la mayor capacidad para hacer una predicción de los niveles de radiación en el período estipulado en la región de Garanhuns. En la investigación, utilizamos datos de los niveles de radiación en el municipio por día para el ciclo bajo análisis, así como información sobre variables como la humedad del aire, la temperatura, las características del viento (dirección, ráfaga y velocidad), entre otros, las cuales se encuentran disponibles en el Instituto Nacional de Meteorología (INMET). Para el análisis comparativo entre los modelos, se utilizaron las pruebas de Dicker-Fuller Aumentado, Shapiro-Wilk, Kruskal-Wallis para verificar la estacionariedad, normalidad y estacionalidad, respectivamente, para los modelos en cuestión. Además, los tipos de modelos que se compararon fueron ARIMA y ARIMAX, que originalmente eran similares, lo que refuerza la importancia de la correcta aplicación de las pruebas y el análisis comparativo. Se descubrió que el mejor modelo a aplicar es ARIMAX, que identifica mejor los ciclos de crecimiento y disminución de los niveles de radiación durante el período estudiado. En cuanto a la previsión, para los próximos diez años, se espera que incluso con la aparición de un comportamiento periódico, la variabilidad aumente. Finalmente, se concluyó que el uso del modelo ARIMAX contribuye a la prevención de enfermedades y a un uso más eficiente e inteligente de la energía solar en Garanhuns.

PalabrasClave:Ajuste, Pronóstico, Radiación, Garanhuns

\begin{abstract}
Looking for analyze to large form the radiation data the last 10 years to Garanhuns city, the time series models classified like more indicated was adjusted, not just the nature for presented information was consider in this study like too the particular interesting in predit the insolation levels for the nexts years in the local, being most loyal possible to presented data shape. To select the most adequate model the purpose the work, we realize tests and comparative analyses for to search which model presented better adjust e consequently the better capacity to realize prevision to insolation levels in the stipulated period in Garanhuns. In the research was utilize the daily data to radiation levels in the city for the analyze cycle and information to others variables like air humidity, temperature, wind characteristics (direction, gust and speed), among others, available by Instituto Nacional de Meteorologia (INMET). For comparative analyze between models, was utilize the tests Dicker-Fuller Increased,Shapiro-Wilk, Kruskal-Wallis, looking verify the stationary, normality and seasonality, respectively, for models in study. Too, the models types compared ARIMA and ARIMAX the similar origin, this reforce the importance to accurate application
\end{abstract}


to tests and comparative analyses. Was constacted the better model applied is the ARIMAX, that identify the cycles of growth and decrease in the levels of radiation during the study period. For forecast, for the next 10 years, it is expected that even with the occurrence of periodic behavior, the variability should increase. Lastly, conclud that use of the ARIMAX model contributes to the prevention diseases and for more efficient and intelligent use of solar energy in Garanhuns.

Keywords: Fit, Forecast, Radiation, Garanhuns.

\section{Introdução}

Nas últimas décadas, o meio ambiente tem sofrido com diversas alterações na sua estrutura, o que faz com que muitos de seus habitantes, em especial os cientistas, se preocupem com os grandes problemas globais do presente e que podem comprometer a qualidade de vida no futuro. A exploração de espécies do mundo animal e a destruição de habitats naturais estão entre os principais fatores que agravam os problemas ambientais ao longo dos anos (BARBIERI, 2017).

Dentre os alvos das pesquisas voltadas aos problemas ambientais, estão as variáveis climáticas. O estudo de tais variáveis se justifica, pois estão diretamente associadas a previsão do clima, cujas projeções são consideradas as mais confiáveis (HAMADA, 2017). A radiação solar encontra-se entre tais fatores, visto que está relacionada a variação sazonal da temperatura (OLIVEIRA et al., 2018).

Níveis de radiação e insolação (número de horas de brilho solar) são elementos interligados e seu estudo se faz necessário, não apenas por fatores climáticos mas também pela possibilidade de utilizar a energia solar como fonte renovável de energia (SANTOS, 2016) e para saúde humana, pois tal variável está interligada a enfermidades dentre as quais destaca-se a ocorrência de câncer de pele com alto número de incidências no Brasil (Instituto Nacional de Câncer -INCA).

Apesar da sua importância, poucos estudos foram realizados no sentido de compreender o comportamento da radiação no Brasil (MEDEIROS, 2018; PENEREIRO, 2017; FRAZÃO et al., 2019; KOZMHINSKY et al., 2018; SILVA et al., 2018 ), dentre as dificuldades encontradas está a extensão territorial do país que aumenta o custo para medição (FUNARI, 2017).

Diante do exposto, o objetivo do projeto consiste em analisar, dentre os modelos ARIMA e ARIMAX, qual apresenta o melhor ajuste para os dados de radiação do município de Garanhuns, e a partir desse modelo, prever os níveis da radiação para os próximos dez anos. 


\section{Fundamentação Teórica}

\section{Séries Temporais}

Seja $Z_{t}=\left\{z_{t} \in \mathbb{R} \mid t=1,2, \cdots, n\right\}$ uma série temporal de um conjunto de observação ordenadas no tempo $t$, sendo $n$ o número de observações. Para que uma determinada série seja classificada como uma série temporal, é necessário que os dados apresentem uma dependência serial entre eles. Segundo a abordagem de componentes não observáveis, as séries temporais podem ser representadas como a combinação de quatro componentes (Mendenhall, 1996): Tendência, Cíclica, Sazonal e Componente do Erro. Pode-se classificar as séries temporais como: estacionárias, não estacionárias e ergódigas, para a confirmação dos componentes e estacionariedade se faz necessário realizar os testes de Dicker-Fuller Aumentado, Shapiro-Wilk e Kruskal-Wallis, logo em seguida realizar a previsão.

\section{Dicker-Fuller Aumentado}

O teste de Dickey-Fuller Aumentado (ADF) investiga a existência ou não da estacionariedade entre variáveis individuais num conjunto de dados. O teste consiste em estimar

$$
\nabla Y_{t}=\alpha+\sum_{i=1}^{p}\left(\theta_{i}-1\right) Y_{t-1}+\sum_{i=1}^{p-1} \sum_{j=i+1}^{p}-\theta_{j} \nabla Y_{t-1}+\gamma T+u_{i}
$$

em que $\alpha, \theta$ e $\gamma$ são os coeficientes a serem estimados pela regressão correspondente a série $Y_{t}$ na primeira diferença no instante $t, u_{i}$ o ruído branco e $T$ a variável que determina a tendência determinística. E pode ser realizado para testar a seguinte hipótese:

$H_{0}$ : A série não é estacionária

$H_{1}$ : A série é estacionária

$\mathrm{Se} H_{0}$ for rejeitado, a estatística calculada de Dicker-Fuller é significativa, então a série é estacionária. Caso $H_{0}$ não seja rejeitada, a estatística calculada não é significativa, e a série é não estacionária (TEY; RADAM, 2011; GAIO; ALVES; PIMENTA JÚNIOR, 2009).

\section{Shapiro-Wilk}

O teste Shapiro-Wilk (SW) (1945) foi desenvolvido para testar a normalidade de uma amostra. O teste é considerado mais poderoso e abrangente na maioria das situações, tornando-se o mais utilizado teste de normalidade devido às boas propriedades de potência 
em comparação com uma ampla gama de testes alternativos. O teste de SW depende da correlação entre os dados fornecidos e seus escores normais correspondentes. A estatística do teste é dada por

$$
W=\frac{\left\{\sum_{i=1}^{n} a_{i} X_{(i)}\right\}^{2}}{\sum_{i=1}^{n}\left(X_{i}-\bar{X}\right)^{2}}
$$

em que $X_{(i)}$ é a i-ésima maior estatística de ordem, $\bar{X}$ é a média amostral e n é o número de observações (MENDES; PALA, 2003; ÖZTUNA; ELHAN; TÜCCAR, 2006; GHASEMI; ZAHEDIASL, 2012).

\section{Kruskal-Wallis}

O teste não paramétrico de Kruskal-Wallis é utilizado para verificação de sazonalidade (PROVENZA; DA SERRA COSTA; DE CARVALHO SILVA, 2018). A sazonalidade nos dados demonstra os padrões de comportamento que acontecem nos períodos de tempos. Sua presença é constatada através da averiguação do gráfico e pelo conhecimento preexistente do comportamento da série temporal. Entretanto, encontra-se algumas séries temporais questionáveis em relação ao componente sazonal (KURUKULASOORIYA; LELWALA, 2014).

Admitindo que as observações foram coletadas em períodos de tempo com espaçamentos iguais, cada coluna $K$ é classificada como uma amostra de tamanho $n_{i}$, assim tem-se

$$
Z_{i j}, j=1, \ldots, k, i=1, \ldots, n_{j} N=\sum_{j=1}^{K} n_{j}
$$

Substituindo as observações $Z_{i j}$ pelos referentes postos ou ordem $R_{i j}$, adquiridos ordenando todas as $N$ observações, sendo $R_{. j}$ o somatório dos postos relacionados à j-ésima amostra, tem-se que

$$
R . j=\sum_{i=1}^{n j} R_{i j}, j=1, \ldots, k
$$

Então a estatística do teste é

$$
T_{1}=\frac{12}{N(N+1)} \sum_{j=1}^{k} \frac{R_{. j}^{2}}{n_{j}}-3(N+1)
$$

As hipóteses consideradas são 
$H_{0}$ : Não existe sazonalidade;

$H_{1}$ : Existe sazonalidade.

Se a estatística do teste for maior ou igual ao valor crítico $T_{1 c}$, então a hipótese nula $\left(H_{0}\right)$ será rejeitada (valor tabelado da $\left.\mathcal{X}^{2}\right)\left(T_{1} \geq T_{1 c}\right.$ ), dado o nível de significância $\alpha$ (MORETTIN; TOLOI, 2004; SIEBEN, 2017).

\section{Modelo ARIMA}

O modelo $\operatorname{ARIMA}(p, d, q)$ representa uma série temporal empregando diferenciação adequadaa um modelo ARMA (uniãodo modelo auto-regressivo (AR) como processo médiasmóveis (MA)), onde 'p' diz respeito ao número de defasagens da série, 'd' é a ordem de integração e 'q' é o número de defasagens dos erros aleatórios (YANG et al., 2017;DA SILVA, 2019).

O ARIMA baseia-se na adaptação dos valores observados, objetivando diminuir a diferenciação dos valores produzidos no modelo e os valores observados. Esse modelo apresenta a possibilidade de caracterizar o comportamento das séries estacionárias e não estacionárias, fornecendo versatilidade para uma variedade de situações (SATO, 2013).O modelo ARIMA é definido como

$$
\Phi(B) \nabla^{d} y_{t}=\Theta(B) \varepsilon_{t}
$$

em que $B$ representa o retardo temporal, $\nabla^{d}$ é o operador de diferenciação, $\Phi(B)$ é um polinômio auto regressivo de ordem $p, \mathrm{e} \Theta(B)$ é um polinômio de média móvel de ordem $q$.

\section{Modelo ARIMAX}

O modelo ARIMAX é uma generalização do modelo ARIMA em que é obtida através da inclusão de variáveis exógenas. Originalmente, o modelo ARIMAX foi proposto por Box e Tiao (1981), com o intuito de estudar o efeito das velocidades de entrada de gás nas concentrações de saída do dióxido de carbono. Inicialmente para determinar um modelo ARIMAX, se faz necessário ajustar o modelo ARIMA univariado com apenas uma única entrada (WILLIAMS, 2001). O modelo ARIMAX é definido como

$$
\Phi(B) \nabla^{d} y_{t}=\mu+\Theta(B) x_{t}+\Theta(B) \varepsilon_{t}
$$

em que $B$ representa o operador de deslocamento temporal para defasagens anteriores ou retardo temporal, $\nabla^{d}$ é o operador de diferenciação $(1-B)^{d}, \Phi(B)=1-\sum_{i=1}^{p} \emptyset_{i} B$ é um 
polinômio auto regressivo de ordem $p, \Theta(B)=1-\sum_{i=1}^{q} \theta_{i} B$ é um polinômio de média móvel de ordem $q$. E $x_{t}$ é uma matriz de variáveis exógenas.

De acordo com Jalalkamali et al., (2015) a vantagem de se trabalhar com o modelo ARIMAX é a inclusão de preditores lineares em sua estrutura, que melhora a precisão da previsão. Segundo Yang et al., (2017) as etapas para obter uma análise e previsão são: (i) Testar a estacionaridade da série de respostas; (ii) Computar as estatísticas que representam as características das séries de respostas, isto é, a função de auto correlação (ACF) e a função de auto correlação parcial (PACF), para estipular os parâmetros $p, \mathrm{~d}$ e $q$; (iii) Estimar os parâmetros desconhecidos do modelo e testara significância, como também as séries residuais;(iv)Verificar as séries de entradas da mesma forma que a série de respostas; (v) Estimar o coeficiente de correlação cruzada entre as séries para estabelecer o aspecto do modelo ARIMAX; (vi) Determinar análises de diagnóstico para averiguar se o modelo condiz com às características dos dados.

\section{Metodologia}

Neste trabalho analisou-se dados das séries climáticas, apresentando níveis de radiação, umidade do ar, temperatura, velocidade do vento, rajada do vento, direção do vento, temperatura de ponto de orvalho, precipitação, pressão atmosférica do município de Garanhuns no período de 2009 a 2018, disponibilizadas pelo Instituto Nacional de Meteorologia (INMET).

Os dados horários disponibilizados pelo INMET foram agrupados para obtenção de medidas diárias, onde no caso da precipitação utilizou-se o acumulado diário e para as demais séries utilizou-se a média diária. Os dados faltantes foram interpolados pela média das últimas 10 observações.

Uma pré-analise foi realizada para as variáveis exógenas com o intuito de não haver problema de multicolinearidade no modelo. Também foi calculada a correlação entre as variáveis e verificou-se se que havia variáveis exógenas com alta correlação e, dentre estas, apenas as mais correlacionadas com a radiação foram incluídas no modelo.

\section{Resultados e Discussão}

Pode-se notar que os dados de radiação do município de Garanhuns se distribuem simetricamente em torno da mediana $1500 \mathrm{Kj} / \mathrm{m}^{2}$, porém a forte incidência de outliers que 
pode ser observada na Figura 1 influencia o comportamento da série de tal maneira que o histograma, como visto na Figura 2, apresenta assimetria positiva em torno da média.

Figura 1: Distribuição de frequência de níveis de radiação em Garanhuns, no período de janeiro de 2009 a dezembro de 2018 .

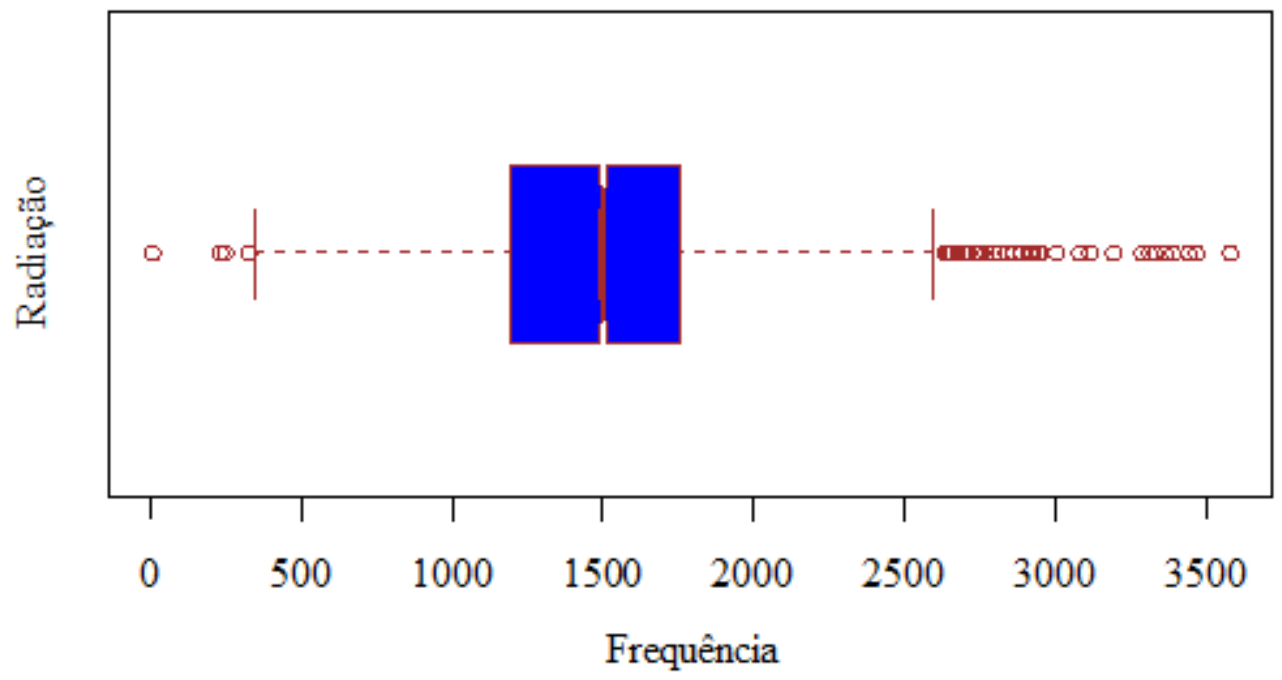

Fonte: Própria (2019).

Figura 2: Densidade de níveis de radiação em Garanhuns, no período de janeiro de 2009 a dezembro de 2018.

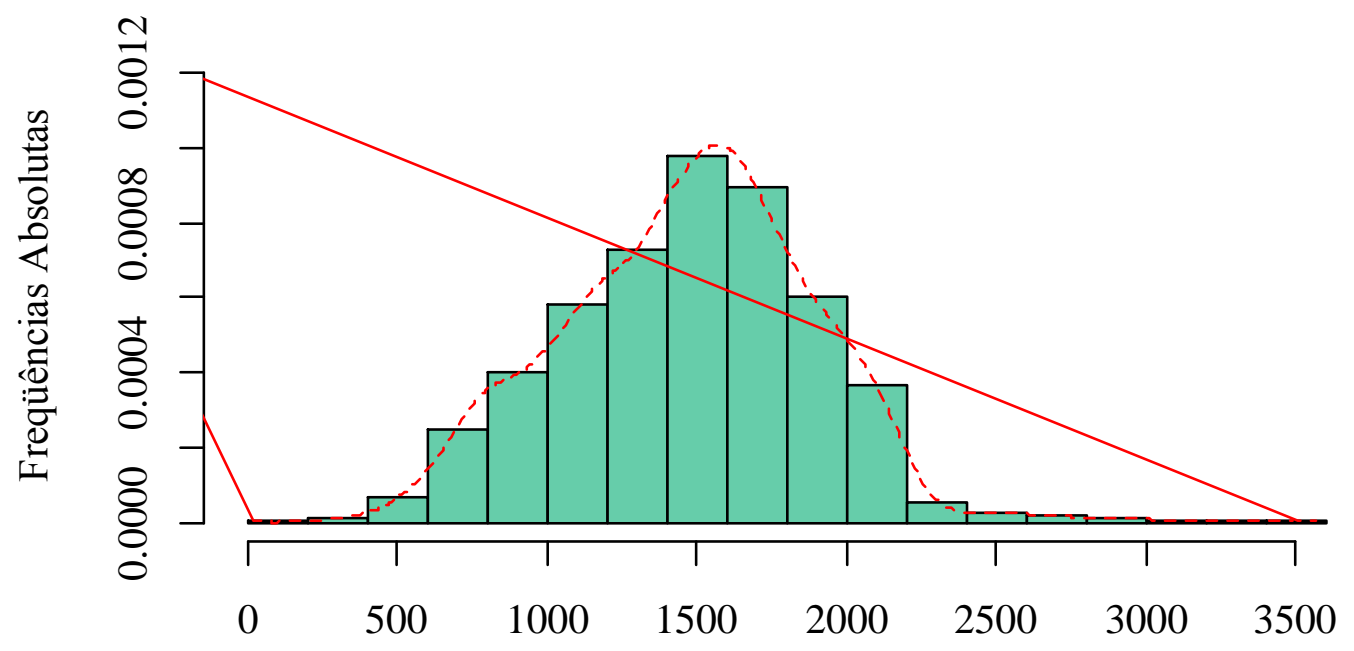

Classes

Fonte: Própria (2019).

Na Tabela 1, estão os resultados da aplicação dos testes de Shapiro-Wilk para verificar a hipótese de normalidade, Dickey-Fuller para testar raiz unitária e, consequentemente estacionariedade e o de Kruskal-Wallis para avaliar a presença de comportamento sazonal. Os 
resultados apresentados confirmam o que analiticamente observa-se nas Figuras 1-2, ou seja, devido a influência dos outliers, que levam a assimetria (característica de distribuições não normais), verifica-se o comportamento de ausência de normalidade dos dados. Os demais testes confirmam propriedades importantes da série de radiação e por meio das hipóteses testadas, conclui-se que a série é estacionaria e não possui comportamento sazonal.

Tabela 1: Resultados obtidos com a aplicação dos respectivos testes, Shapiro-Wilk, Dicker-Fuller e KruskalWallis para a cidade de Garanhuns/PE, no período de janeiro de 2009 a dezembro de 2018.

\section{Município}

\section{Testes}

\begin{tabular}{lccc}
\cline { 2 - 4 } \multicolumn{1}{c}{ Garanhuns } & Shapiro-Wilk & Dicker-Fuller & Kruskal-Wallis \\
\hline Estimativas & 0,987 & $-5,918$ & 28,919 \\
p-valor & $<0,01$ & $<0,01$ & 0,5218 \\
\hline
\end{tabular}

Fonte: Própria (2019).

A Figura 3 apresenta os gráficos LAG's da série temporal da radiação em Garanhuns, em que o ponto corresponde ao valor da série temporal no tempo t versus o valor no tempo $\mathrm{t}-1$, t-2, t-3 e t-4, usados para exibir eventuais correlações entre valores consecutivos. Embora não exista evidência de um padrão linear claro, isso não implica que a estrutura temporal é completamente desordenada e aleatória. Contudo, os gráficos apresentaram uma correlação alta, conforme Hopkins (2000).

Figura 3: Gráfico LAG’s da série temporal da radiação em Garanhuns/PE para t versus o valor no tempo t-1, t-2, 

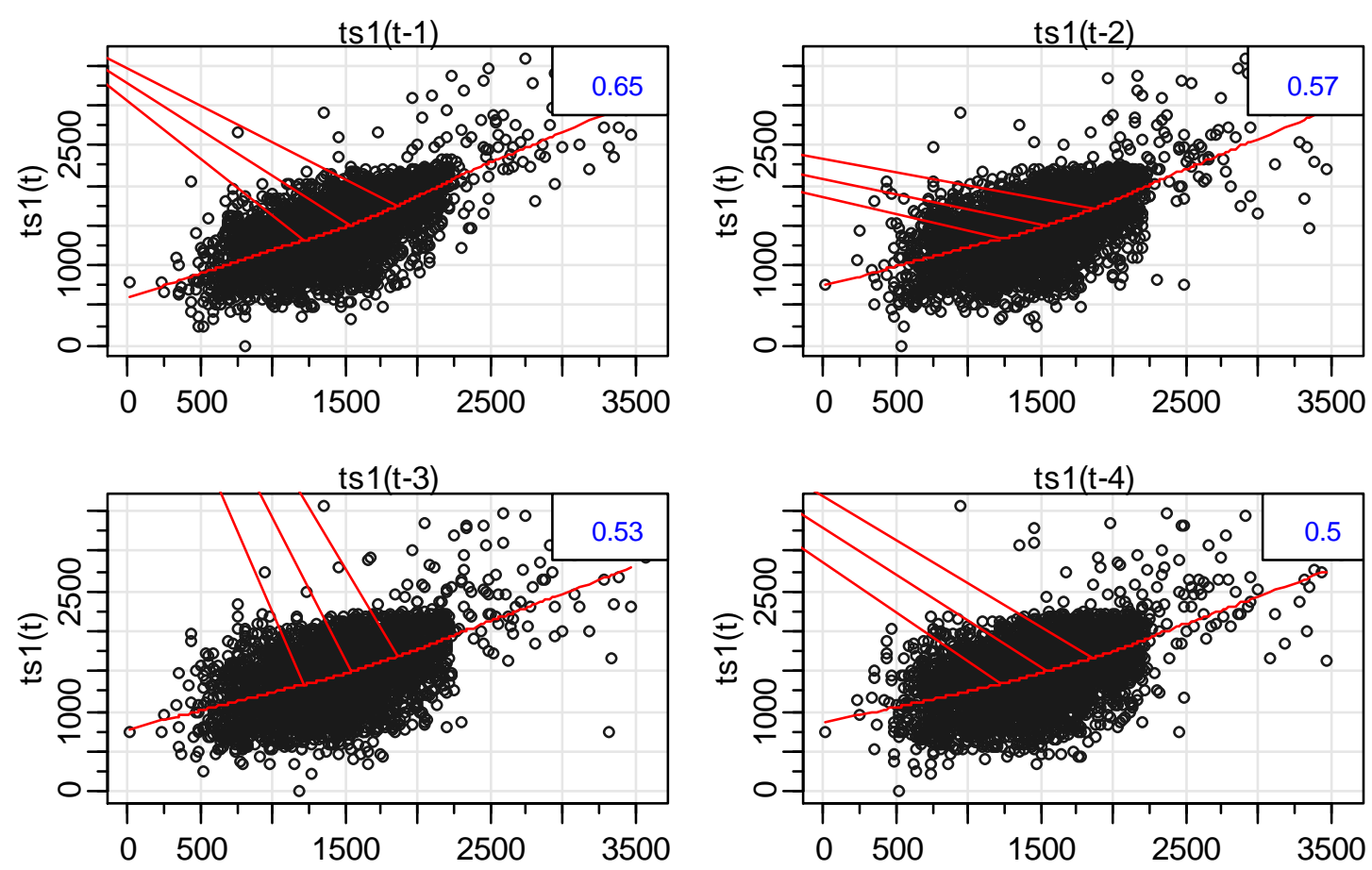

Fonte: Própria (2019).

A modelagem da radiação solar é uma ferramenta importante para estudar o potencial de geração de energia de um local. Couto, Oliveira e Braga (2016) utilizaram médias móveis e projeção exponencial no intuito de realizar projeções de radiação no Ceará e avaliar sua capacidade como fonte de energia renovável. Com este mesmo intuito, para a cidade de Garanhuns, neste trabalho realizou-se duas modelagens para a radiação: a primeira utilizando o modelo ARMA e a segunda utilizando o modelo ARMAX, que trata-se de uma extensão do modelo anterior onde é incluído o efeito de variáveis exógenas, para melhor modelar a variável e de forma a buscar-se diminuir os erros de previsão.

A característica não linear da radiação torna mais difícil encontrar um modelo que capture este comportamento. Neste sentido, Baierle et al. (2019) utilizaram o método de mínimos quadrados com grau polinomial diferente para realizar o ajuste. Através das abordagens, considerando os modelos ARMA e ARMAX, na Figura 3 verifica-se que o modelo que melhor se adapta é o $\operatorname{ARMAX}(1,2)$, que captura a dinâmica do fenômeno que periodicamente cresce e decresce apesar de não ter se encontrado característica sazonal significativa e, além disso, o modelo também ajusta-se bem ao conjunto teste apresentando boas estimativas. No que se refere à previsão, espera-se que para os próximos dez anos o comportamento periódico continue ocorrendo, porém pode-se notar que a variabilidade anual 
deve aumentar ocorrendo picos mais elevados nos períodos anuais que contém radiação máxima.

Figura 4:Modelagem utilizando os Modelos ARMA (a) e ARMAX (b) da série temporal da radiação em Garanhuns/PE, no período de janeiro de 2009 a dezembro de 2018.
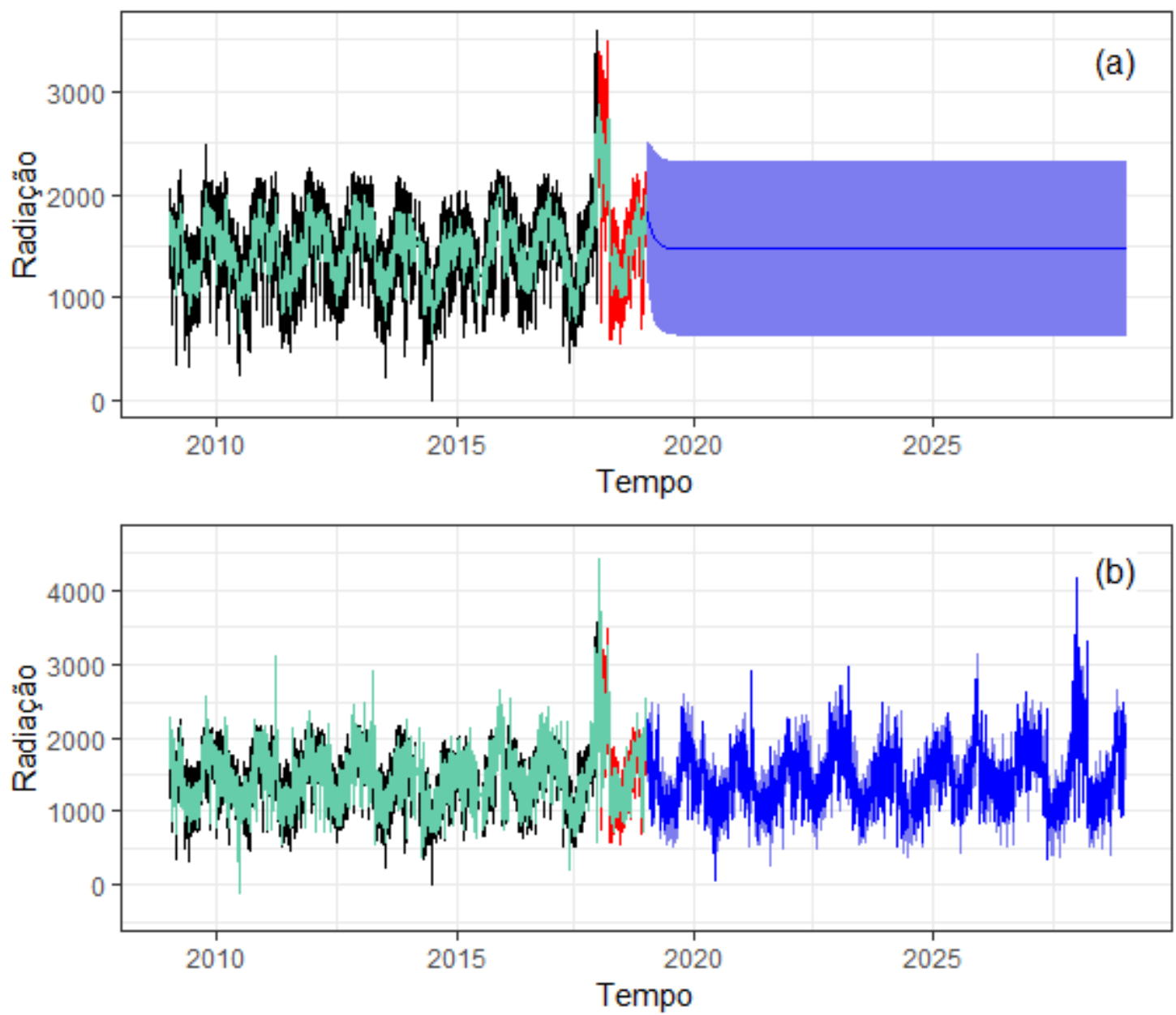

series

- Conjunto de teste — Previsão — Série original — Valores ajustados

Fonte: Própria (2019).

Em relação a medidas de eficiência e bondade do ajuste, como apresentado na Tabela 2, foi identificado que a utilização das modelagens com a inserção de variáveis exógenas proporciona melhores desempenhos para os ajustes aos dados observados em comparação aos outros modelos testados nesta pesquisa, isto é, no município de Garanhuns o uso do modelo ARMAX possui um desempenho melhor do que ARMA. Essa afirmação é baseada no fato que o modelo ARMAX apresentou uma redução da variável MAPE de aproximadamente $13,148 \%$, valor inferior ao adquirido na modelagem ARMA cujo valor é de aproximadamente 22,733\%. Nota-se ainda que para as demais medidas, o modelo ARMAX apresentou as 
menores medidas de acurácia, o melhor desempenho do modelo deve ter ocorrido devido a inclusão de variáveis exógenas que influenciam a dinâmica da radiação. Como exemplo, temse a temperatura do ar que em Macêdo ET AL. (2016) é utilizada numa modelagem matemática para estimar a radiação.

Tabela 2: Medidas comparativas de eficiência dos modelos de Series Temporais para o município de Garanhuns/PE, no período de janeiro de 2009 a dezembro de 2018.

\begin{tabular}{ccccc}
\hline \multirow{2}{*}{ Modelos } & \multicolumn{4}{c}{ Medidas de Eficiência } \\
\cline { 2 - 5 } & ME & RMSE & MAE & MAPE(\%) \\
\cline { 2 - 5 } ARMA & 0,2742 & 302,48 & 237,01 & 22,733 \\
ARMAX & $-0,0249$ & 185,65 & 137,89 & 13,148 \\
\hline
\end{tabular}

Fonte: Própria (2019).

O melhor modelo sugerido pelo critério AIC para o município de Garanhuns foi ARMA(1,2)X (Temperatura do ar, Temperatura do ponto de Orvalho, Pressão Atmosférica, Direção do Vento, Precipitação). A Tabela 3 apresenta os coeficientes do melhor modelo referente ao município de Garanhuns. As variáveis Temperatura do ar, Pressão Atmosférica e Vento direção têm uma influência positiva significativa para os próximos períodos. De modo análogo, as variáveis Temperatura do ponto de orvalho, Velocidade do vento e Precipitação têm influência negativa significativa para a variável resposta.

Tabela 2:Estimação dos parâmetros do ARMAX para o município de Garanhuns/PE, no período de janeiro de 2009 a dezembro de 2018.

\begin{tabular}{lcccc}
\hline \multicolumn{1}{c}{ Modelo } & Estimativa & ErroPadrão & Estatística & p-valor \\
\hline AR(1) & 0,793 & 0,050 & 15,747 & $<0,001$ \\
MA(1) & $-1,548$ & 0,059 & $-26,355$ & $<0,001$ \\
MA(2) & 0,567 & 0,053 & 10,746 & $<0,001$ \\
Temperatura do ar & 195,182 & 4,495 & 43,415 & $<0,001$ \\
Temperatura do ponto de & $-198,932$ & 4,166 & $-47,748$ & $<0,001$ \\
orvalho & & & & \\
Pressãoatmosférica & 17,201 & 3,660 & 4,699 & $<0,001$ \\
Velocidade do Vento & $-10,835$ & 5,547 & $-1,953$ & 0,051 \\
Vento direção & 0,459 & 0,216 & 2,124 & 0,033
\end{tabular}


Precipitação

Fonte: Própria (2019).

Avaliando os resíduos do modelo na Figura 5, observa-se que os resíduos não seguem distribuição Normal, pois os pontos que se localizam nas extremidades se desviam da reta ajustada. No entanto, os resíduos se comportam como uma sequência de variáveis aleatórias independentes, identicamente distribuídas com média zero e variância constante. Portanto, esses modelos conseguem captar o nível e inclinação, mas não as flutuações nas extremidades, logo, os modelos são bons em predizer observações em pequenos intervalos de tempo.

Figura 5: Análise residual do modelo ARIMAX para o município Garanhuns/PE, no período de janeiro de 2009 a dezembro de 2018.

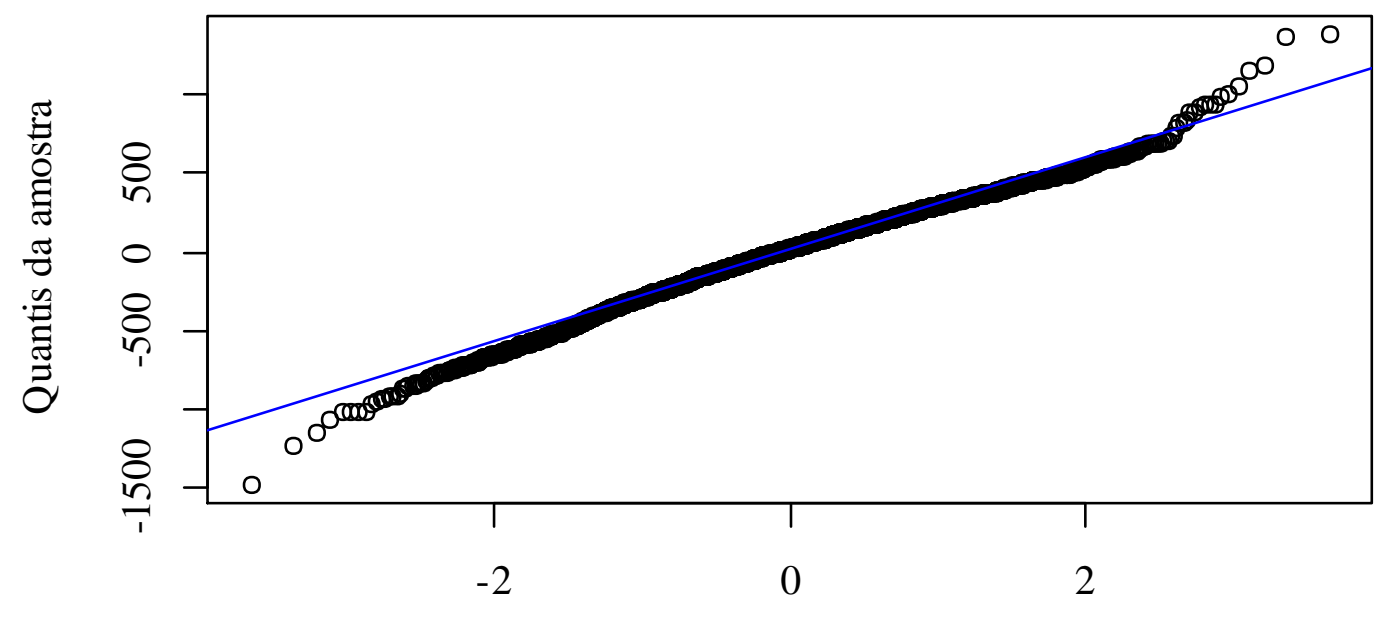

Quantis teoricos

Fonte: Própria (2019).

\section{Agradecimentos} financeiro.

Os autores agradecem às agências de CAPES, CNPq, FACEPE e UFRPE pelo suporte

\section{Conclusões}

Como vimos, o modelo ARIMAX conseguiu captar o comportamento da radiação global para o município de Garanhuns, onde os valores estimados do modelo acompanharam a série temporal original. Portanto, tal ferramenta é viável para auxiliar na tomada de decisões, tanto das doenças causadas pelo excesso de exposição ao sol quanto dos processos de transformação de energia solar em energia utilizável, em termos das horas/dias de radiação na 
cidade de Garanhuns. Dessa forma, é possível ajudar nas políticas de saúde pública, incentivando ainda mais a utilização de protetores solares nas épocas em que o quantitativo de horas/dias de radiação fosse maior. Ainda, houve uma contribuição no desenvolvimento de pesquisas sobre a transformação de energia solar em energia utilizável.

\section{Referências}

ABC do Câncer. Abordagens básicas para o controle do câncer. Ministerio da Saúde, Instituto Nacional de Câncer Jose Alencar Gomes da Silva (INCA); organização Mario Jorge Sobreira da Silva. 3 ed. rev. atual. Rio de Janeiro: Inca; 2017. 108 p. Disponível em: http://www1.inca.gov.br/inca/Arquivos/livro-abc-3ed-8a-prova.pdf

BAIERLE, E., MARTINS, M. T. F., RODRIGUES, M. B., \& BLASS, L. (2019). Estudo da curva de radiação solar no município de bagé utilizando métodos numéricos. Anais do Salão Internacional de Ensino, Pesquisa e Extensão, v. 10, n. 2, 2019.

BARBIERI, J. C. Gestão ambiental empresarial. Editora Saraiva, 2017.

COUTO, H. J. B.; OliveIRA, R. A. E.; BRAGA, P. F. A. Previsão de Radiação Solar Incidente no Estado do Ceará-Brasil. HOLOS, v. 7, p. 218-230, 2016.

DA SILVA, C. A. G. Análise da previsão do preço do etanol hidratado no estado de São Paulo: uma aplicação do modelo arima/Analysisoftheforecastingofthehydratedethanolprice in thestateof São Paulo:anapplicationofthearimamodel. BrazilianJournalofDevelopment, v. 5, n. 10, p. 1776317778, 2019.

DA SILVA, M. P. A importância do uso de epi na prevenção de acidentes de trabalho na indústria madeireira. FACIDER-Revista Científica, v. 3, n. 3, 2013.

DE MACÊDO, K. G., OLIVEIRA, J. B., ARRAES, F. D. D., TORRES, W. L. V., \& JUNIOR, J. C. L. (2016). Nota técnica: Estimativa da radiação solar global com dados de temperatura do ar em seis cidades do estado do ceará. Revista Engenharia na AgriculturaReveng, v. 24, n. 1, p. 68-75, 2016.

DE MEDEIROS, R. M. Insolação decadal para Recife-PE, BRASIL. Revista GeograficaAcademica, v. 12, n. 2, p. 124-137, 2018.

DE OLIVEIRA, M. J.; CARNEIRO, C. D. R.; VECCHIA, F. A. S.; de MELLO BAPTISTA, G. M. Ciclos climáticos e causas naturais das mudanças do clima. TerraeDidatica, v. 13, n. 3, p. 149-184, 2019.

FRAZÃO, F. B.; FERREIRA, L. K. S.; FRAZÃO, R. H. N.; LOUZEIRO, N. M. Riscos ocupacionais e medidas de proteção dos trabalhadores identificados em uma piscicultura no município de SantaRita-MA. Revista Brasileira de Engenharia de Pesca, v. 12, n. 1, p. 50-61, 2019. 
FUNARI, F. L.; TARIFA, J. R. Insolação, radiação solar global e radiação líquida no Brasil. Instituto Geologico, v. 38, n. 2, p. 49-83, 2017.

GAIO, L. E.; DE FREITAS ALVES, K. L.; PIMENTA JÚNIOR, T. O mercado acionário brasileiro do novo milênio: um teste de eficiência. BBR-Brazilian Business Review, v. 6, n. 3, p. 231-246, 2009.

GHASEMI, A.; ZAHEDIASL, S. Normalitytests for statisticalanalysis: a guide for nonstatisticians. Internationaljournalofendocrinologyandmetabolism, v. 10, n. 2, p. 486, 2012.

HAMADA, E.; DE OLIVEIRA, E.; CHARLOTTE, E.; LANDAU, S. M. C. N.; GHINI, R. Impacto das mudanças climáticas sobre a distribuição geográfica das ferrugens do milho. Aquecimento Global e Problemas Fitossanitários, p. 221, 2017.

HOPKINS, W.G. Correlationcoefficient: a new view of statistics. Disponível em: http://www.sportsci. org/resource/stats/correl.html, 2000. Acesso em: 16fevereiro. 2019.

JALALKAMALI, A.; MORADI, M.; MORADI, N. Applicationofseveral artificial intelligencemodelsand ARIMAX model for forecastingdroughtusingtheStandardizedPrecipitation

Index. Internationaljournalofenvironmentalscienceandtechnology, v. 12, n.4, p. 1201-1210, 2015.

KOZMHINSKY, M.; DE MEDEIROS, R. M.; DE HOLANDA, R. M.; SILVA, V. D. $P$. Average insolation interpolated by the krigagem method for the state of Pernambuco-B razil. JournalofHyperspectral Remote Sensing v. 8, n. 2, p. 78-84, 2018.

KURUKULASOORIYA, N.; LELWALA, E. Time series behaviorofburgeoninginternationaltouristarrivals in Sri Lanka: The postwarexperience. RuhunaJournalof Management andFinance, v. 1, n. 1, p. 1-14, 2014.

MENDES, M.; PALA, A. Type I $\quad$ error rate andpowerofthreenormalitytests. PakistanJournalofInformationand Technology, v. 2, n. 2, p. 135-139, 2003.

MENDENHALL, W.; SINCICH, T.; BOUDREAU, N. S. A second course in statistics: regression analysis. Upper Saddle River, NJ: Prentice Hall, 1996.

MOREtTIN, P. A.; TOLOI, C. M. C. Análise de SériesTemporais. São Paulo: Edgard Blucher LTDA, 2004, 535 p.

ÖZTUNA, D.; ELHAN, A. H.; TÜCCAR, E. Investigationof four differentnormalitytests in termsoftype 1 error rate andpowerunderdifferentdistributions. TurkishJournalof Medical Sciences, v. 36, n. 3, p. 171-176, 2006.

PENEREIRO, J. C.; MACCHERI, N. A.; BADINGER, A. Análises da insolação sobre a temperatura média e a precipitação pluvial em cidades brasileiras por meio de 
tendências em séries anuais. Revista GeograficaAcademica, v. 11, n. 2, p. 5-23, 2017.

PROVENZA, M. M.; DA SERRA COSTA, J. F.; DE CARVALHO SILVA, L. Análise de dados e previsão de séries temporais do homicídio doloso no Estado do Rio de Janeiro entre 2001 e 2016. Produção em Foco, v. 8, n. 2, 2018.

SANTOS, M. A. dos. Dimensionamento e retorno de investimento de geração de energia solar residencial: um estudo de caso no município de Lagoa Santa-MG. 2016. 44 p. Monografia (Especialização em Mudanças Climáticas, Projeto Sustentável e Mercado de Carbono) - Universidade Federal do Pará, Curitiba,2016.

SATO, R. C. Gerenciamento de doenças utilizando séries temporais com o modelo ARIMA. Einstein (Sao Paulo), v. 11, n. 1, p. 128-131, 2013.

SIEBEN, E. Modelos Para Previsão em Séries Temporais: A Metodologia Box e Jenkins na Prática. TCC, 2017.

SILVA, V. N.L. Modelagem de dados climáticos e socioeconômicos em municípios do estado de Pernambuco utilizando análise de componentes principais (ACP). 2018. 81 p. Dissertação (Desenvolvimento de Processos Ambientais) - Universidade Católica de Pernambuco, Recife, 2017.

TEY, J. Y. S.; RADAM, A. Demandpatternsof rice imports in Malaysia:Implications for food security. Food Security, v. 3, n. 2, p. 253-261, 2011.

TIAO, G. C.; BOX, G. E.P. Modelingmultiple time series withapplications. Journalofthe American StatisticalAssociation, v. 76, n. 376, p. 802-816, 1981.

WILLIAMS, B. M. Multivariatevehiculartrafficflowprediction: evaluationof ARIMAX modeling. TransportationResearch Record, v. 1776, n. 1, p. 194-200, 2001.

YANG, M.; XIE J.; WANG, C.; YE, Z. Applicationofthe ARIMAX ModelonForecastingFreewayTrafficFlow. In: CICTP 2017: TransportationReformandChange-Equity, Inclusiveness, Sharing, andInnovation. Reston, VA: American Society of Civil Engineers, 2018. p. 593-602. 